\title{
Coulisses
}

Revue de théâtre

4 | Été 1991

Varia

\section{Le rire amer du metteur en scène}

Jean-Yves Debreuille

\section{(2) OpenEdition}

Journals

Édition électronique

URL : http://journals.openedition.org/coulisses/1676

DOI : $10.4000 /$ coulisses. 1676

ISSN : 2546-9460

\section{Éditeur}

Presses universitaires de Franche-Comté

\section{Édition imprimée}

Date de publication : 1 juin 1991

Pagination : $14-20$

ISSN : 1150-594X

\section{Référence électronique}

Jean-Yves Debreuille, «Le rire amer du metteur en scène », Coulisses [En ligne], 4 | Été 1991, mis en ligne le 04 juillet 2017, consulté le 23 octobre 2019. URL : http://journals.openedition.org/coulisses/ 1676 : DOI : $10.4000 /$ coulisses. 1676

Ce document a été généré automatiquement le 23 octobre 2019

Coulisses 


\section{Le rire amer du metteur en scène}

\section{Jean-Yves Debreuille}

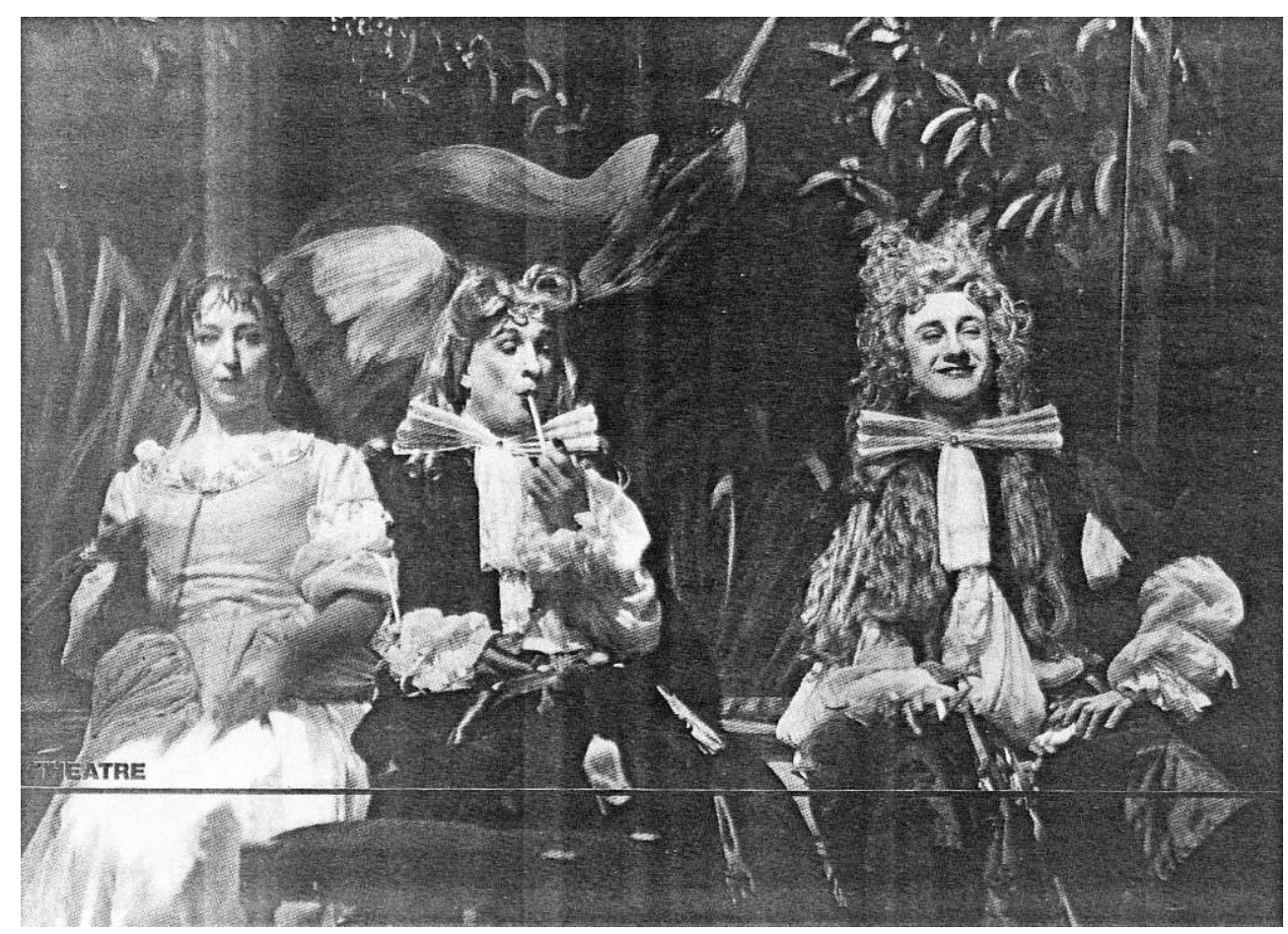

Photo P. Perrin

Le théâtre dans le théâtre est à la mode, et légitimement : au temps du cinéma et de la vidéo, le théâtre sera toujours battu dans l'exactitude de la représentation du réel, ou de ce que nous croyons pouvoir nommer ainsi. Ce qui le rend précisément irremplaçable, c'est le tremblé dû aux aléas quotidiens de la représentation, l'aventure chaque soir renouvelée de comédiens vivants, la difficulté même à montrer et à faire croire, la mise en question vertigineuse des frontières entre le vrai et le faux. Si le XVII ${ }^{\mathrm{e}}$ siècle est l'âge d'or du théâtre, c'est qu'il est celui de la théâtralité : la cour est, par l'étiquette et les cérémonies qui la rythment, un théâtre, on y joue du théâtre, les "divertissements royaux» font collaborer auteurs dramatiques et musiciens à des 
mises en scène dont les courtisans sont tour à tour acteurs et spectateurs. C'est donc tout naturellement que Molière introduit dans telle ou telle de ses pièces (Le Bourgeois gentilhomme, Le Malade imaginaire...) une mascarade qui se prolonge en ballet, qu'il fait d'une pièce le commentaire d'une autre (La Critique de l'Ecole des femmes), qu'il joue de déguisements qui superposent des rôles aux rôles (Dom Juan). Et s'il n'y a apparemment rien de tout cela dans Le Misanthrope, il est tout de même constant que le sujet de la pièce est la manière dont on doit vivre en société, et que le personnage principal est un homme qui entend y régir les comportements. Quand on songe que ce rôle était tenu par le chef de troupe, on ne peut que constater que la même personne prétendait diriger les acteurs du lieu réel (la scène) aussi bien que du lieu virtuel (le monde). On s'aperçoit alors que le texte est riche en indications des deux ordres, et que sa dynamique est purement théâtrale, puisque constituée par la tentative et l'échec d'une mise en scène.

2 Si la première scène de la pièce est assez interminable, c'est qu'elle constitue le programme détaillé du metteur en scène que veut être Alceste. Certes, il proclame sa haine générale de «tous les hommes ", mais les «mouvements soudains / De fuir dans un désert l'approche des humains " ne le prennent que "parfois ", et ses projets sont beaucoup plus sociaux. "Je veux qu'on me distingue », affirme-t-il, et le fait d'être seul contre tous n'est pas pour déplaire à ce chevalier de la vérité, qui ne peut s'imaginer autrement qu'en point de mire de l'humanité entière. Dans ces conditions, même une défaite ne peut être que glorieuse, puisqu'elle transforme " aux yeux de l'univers » sa victime en martyr d'une cause superbe :

Je verrai, dans cette plaiderie,

Si les hommes auront assez d'effronterie,

Seront assez méchants, scélérats et pervers,

Pour me faire injustice aux yeux de l'univers.

"Aux yeux de l'univers": cela vaut bien de "rompre en visière à tout le genre humain»! Alceste va donc construire la trame d'une pièce à sketches, qu'est par certains côtés Le Misanthrope, en programmant un certain nombre d'affrontements. Affrontements individuels d'abord, dont il évoque quelques exemples avec Philinte, et qui consisteront à dire à chacun son fait, et à " n'épargner personne sur ce point » : à la vieille Émilie qu'elle n'a plus un âge à faire la coquette, à Dorilas que ses discours d'autosatisfaction assomment... En fait, c'est Oronte qui va se présenter le premier, et qui va faire à propos de son sonnet l'expérience de cette nouvelle franchise. Affrontement des pouvoirs sociaux ensuite : c'est le fameux procès pour lequel Alceste refuse de visiter les juges comme le réclament les usages et la prudence... et dont on lui annoncera effectivement la perte à la dernière scène de l'acte IV. Affrontement de celle qu'il aime enfin, dont il ne doute pas de triompher, tant il est sûr que sa flamme « De ces vices du temps pourra purger son âme ».

4 C'est à ce dernier programme qu'il va mettre le plus de soin. Pour cela, il va réclamer d'abord la maîtrise de la scène, tentant d'obtenir de Célimène au début de l'acte II qu'elle ferme sa porte aux visiteurs. Ayant échoué sur ce point, il va imposer sa présence, critiquant ouvertement les marquis et celle qui les reçoit dans la scène 4 de l'acte II, et s'affirmant comme le maître du lieu :

Sortez quand vous voudrez, Messieurs ; mais j'avertis

Que je ne sors qu'après que vous serez sortis.

5 Sur ce point non plus, il n'aura pas totalement gain de cause, quoique son temps de présence scénique sur l'ensemble de la pièce soit impressionnant. Mais il va tenter de 
réaliser autrement son désir d'être maître du jeu, en montant une machination qui est un véritable théâtre dans le théâtre : à la scène 2 de l'acte II, il va demander à Eliante de le "venger » en acceptant "son cœur ", c'est-à-dire de jouer avec lui la comédie de l'amour pour dépiter Célimène. Puis, dès la scène suivante, nouvelle proposition de jeu, à laquelle il ne croit guère plus : celle de l'innocence de Célimène :

Efforcez-vous ici de paraître fidèle

Et je m'efforcerai, moi, de vous croire telle.

En fait, depuis un moment, le metteur en scène s'affole, et son programme perd toute cohérence. S'il réaffirme au début de l'acte $\mathrm{V}$ sa volonté de perdre son procès, il n'attend plus comme spectateur du «bon droit maltraité » que la postérité, et à la scène suivante, il contracte alliance avec Oronte, son pire ennemi, pour obliger Célimène à dévoiler ses propres sentiments. Il parle toujours haut et fort, mais il ne maitrise plus l'enchaînement d'événements qu'il subit de plus en plus, même s'il tente de les réorienter à son profit. Le metteur en scène se retrouve mis en scène.

7 Il est d'ailleurs un point qu'Alceste n'a jamais parfaitement maîtrisé, c'est sa propre présence scénique. Il suffit qu'il veuille être seul pour qu'aussitôt on s'impose à lui. Philinte ne le « laisse » pas comme il le lui demande au début de la scène 1 de l'acte I, pas plus qu'à la scène 3 du même acte, Célimène lui ordonne de « demeurer » contre sa volonté à la scène 3 de l'acte II (jusqu'à ce que, se souvenant de son principe de contradiction, elle lui permette de sortir, s'assurant ainsi qu'il restera), Philinte refuse de l'abandonner « dans (son) petit coin sombre, avec (son) noir chagrin » à la scène 1 de l'acte $V$, et quand au terme de la pièce il envisage enfin de se retirer définitivement, c'est encore cet ami trop sociable qui décide d'entraver cette résolution. Inversement, si Alceste décide de rester, tout se ligue pour le gêner par une présence importune (c'est le cas des marquis qui interrompent son « tête à tête » avec Célimène à la scène 2 de l'acte II), ou pis encore pour l'exclure : à la fin de l'acte II, c'est un garde qui vient le chercher de la part des Maréchaux; au terme de l'acte III, c'est Arsinoë qui l'enlève ; à la fin de l'acte IV, c'est son valet Du Bois qui vient l'avertir qu'« il faut d'ici déloger sans trompette ».

D'ailleurs, même quand il est metteur en scène efficace, le misanthrope n'est pas nécessairement souverain, dans la mesure où ses initiatives programmatrices ne proviennent pas tant de sa volonté propre que des excès dans lesquels les autres le poussent. Dès la scène 1 de l'acte I, c'est Philinte qui le provoque :

Quoi ! Vous iriez dire à la vieille Émilie

Qu'à son âge il sied mal de faire la jolie [...] ?

9 Alceste ne peut que répondre "Sans doute", et quand il affirme ensuite que son dessein " est de rompre en visière à tout le genre humain ", il obéit moins à un calcul réfléchi qu'à l'état d'exaspération dans lequel l'a poussé Philinte, véritable metteur en scène en l'occurrence. D'ailleurs, tout le monde connait l'unique principe qui le meut, formulé par Célimène : «Et ne faut-il pas bien que Monsieur contredise ?» (v. 679). À partir du moment où ses adversaires - ou ses amis, s'ils ne sont les mêmes -, ont compris cela, ils peuvent le manipuler à leur guise.

10 Mais il est une force encore bien plus sûre pour mener Alceste là où il ne veut point aller, c'est son amour pour Célimène. Cette dernière n'est pas la seule à l'utiliser : si Arsinoë réussit à l'attirer chez elle, en espérant bien lui « offrir de quoi (le) consoler " (v. 1132), c'est parce qu'elle lui promet une preuve de l'infidélité de celle qu'il aime. Oronte lui-même, son pire ennemi, réussit à se l'allier quand il accule Célimène à 
« choisir » (v. 1603). Mais bien évidemment, c'est cette dernière qui joue en virtuose de la passion qu'elle suscite, réussissant dans la scène 3 de l'acte IV à transformer Alceste d'accusateur en suppliant, et à faire que contre tous ses principes, il préfère un mensonge peu crédible aux rigueurs de la vérité, reconnaissant lui-même le dédoublement qu'il subit en la circonstance :

$\mathrm{Ah}$ ! que vous savez bien ici, contre moi-même

Perfide, vous servir de ma faiblesse extrême.

11 Et finalement, à la scène 4 de l'acte $\mathrm{V}$, ce contempteur du genre humain, celui qui n'aime rien tant que le rôle d'accusateur et de dénonciateur du mensonge, restera muet au grand procès de Célimène, comme hors du débat où d'autres jouent les premiers rôles :

Hé bien! je me suis tu, malgré ce que je voi,

Et j'ai laissé parler tout le monde avant moi.

Cruel accès de lucidité... et de modestie forcée. Celui qui se voyait martyr de sa noble cause «aux yeux de l'univers» (v. 200) réalise qu'il n'est que ce que lui avait prédit Philinte, «Et qu'un si grand courroux contre les mœurs du temps / (le) tourne en ridicule auprès de bien des gens" (v. 107-108). À la fin de l'acte II, après avoir été impitoyablement et publiquement portraituré par Célimène, puis sorti du salon où paradaient ses rivaux par le garde des Maréchaux, il avait déjà pressenti que sa situation était plus risible qu'héroïque :

Par la sangbleu! Messieurs, je ne croyais pas être

Si plaisant que je suis

(v. 773)

13 Désormais, il entend ce qu'il est dans les paroles des autres : un objet de mépris, que l'on abandonne à son insignifiance. «[...] Je ne fais plus obstacle à votre flamme ", annonce Oronte à celui qu'Arsinoë nomme délicatement «le rebut de Madame». Tous s'en vont vers un lieu plus brillant, lieu visible où Alceste rêvait, quoi qu'il en dise, de lancer ses anathèmes et de régir ses semblables par la mauvaise conscience. On n'échappe pas si facilement à l'idéal théâtralisé de la cour et des salons.

De fait, Alceste n'a été metteur en scène que d'une chose, c'est de sa propre exclusion. Ce qu'il réalise peu à peu, et parfaitement, c'est ce qu'il présentait au début comme une velléité : sa retraite au désert. C'est dans ce qui apparaissait comme ses excès de langage qu'est la vérité de sa conduite. N'avait-il pas annoncé à Célimène dès leur première entrevue, au début de l'acte II :

"Tôt ou tard nous romprons indubitablement?

En fait, il construit méthodiquement son exclusion, d'abord du théâtre plus vaste qu'est la société politique : à Oronte qui lui proposait de faire pour lui «quelque ouverture » auprès du Roi, à Arsinoë qui s'offre à « remuer des machines " pour lui obtenir « une charge à la cour ", il répond de même :

Et sur quoi pourrais-je en rien prétendre?

Quel service à l'Etat est-ce qu'on m'a vu rendre?

16 Cette affirmation de son inutilité est grave. Elle ne contredit pas seulement ses velléités de réforme du fonctionnement social, mais même l'intérêt exemplaire de son martyre. Elle nie même sa visibilité : non seulement il disparaîtra, mais nul ne s'en apercevra.

17 Plus subtilement, mais plus gravement encore, il mine les valeurs qu'il entend faire triompher. Il attend de Célimène une sincérité absolue : 
Mais qui m'assurera que, dans le même instant,

Vous n'en disiez peut-être aux autres tout autant.

(II, 1). de vingt ans » que la " solitude » effraie si fort, et qui a tant fait pour s'en préserver, qu'il va proposer de le suivre dans son désert! Autant dire qu'il est le metteur en scène enfin comblé de ce dernier rejet. L'a-t-il provoqué inconsciemment pour mettre en relief son absolue solitude? Ses dernières paroles pourraient le laisser croire : il est enfin «trahi de toutes parts », il réalise enfin la démonstration expérimentale que «tous les hommes » sont "méchants et malfaisants», ou au moins "aux méchants complaisants ». Mais il n'a pas le dernier mot: celui-ci appartient à son ami Philinte, qui s'empresse d'avenir que la société va tout faire pour l'empêcher de réaliser son ultime programme. Même ailleurs, sur une autre scène, en " un endroit écarté ", son autonomie dérangerait. Sa conduite doit lui être dictée, et elle le sera par tous les moyens :

Allons employer toute chose

Pour rompre le dessein que son cœur se propose.

21 «Il y a un tragique moderne : c'est une espèce de grand volant qui tourne et qui n'est pas dirigé par la main ", écrit Aragon. C'est de ce tragique-là que le personnage installé sur scène par Molière fait l'expérience. Expérience d'homme de théâtre avant tout: rien de plus facile que de présenter l'enchevêtrement des destinées, mais rien de plus difficile que de prétendre les diriger. Le théâtre comme la société vit de fausse monnaie que les acteurs échangent sans y croire vraiment, mais sans la dénoncer non plus, car alors le commerce et le jeu cesseraient. Alceste est celui qu'on doit exclure parce qu'il les rendrait impossibles, et exclure en riant, pour conjurer toute tentation de le prendre au sérieux. Heureusement, il se charge de s'exclure lui-même, il suffit de le laisser faire, et de le bousculer un peu au dernier moment pour que ne lui reste pas même la gloire de l'avoir fait. «Je ne croyais pas être / Si plaisant que je suis. » D'après Brossette, « Molière, en récitant cela, l'accompagnait d'un rire amer ». A tout metteur en scène du Misanthrope, à tout comédien jouant Alceste, de retrouver, ou d'inventer, ce rire. 